\title{
Distributed multi-view multi-target tracking based on CPHD filtering
}

\author{
Guchong Li, Giorgio Battistelli, Luigi Chisci, Wei Yi, and Lingjiang Kong
}

\begin{abstract}
This paper addresses distributed multi-target tracking (DMTT) over a network of sensors having different fieldsof-view (FoVs). Specifically, a cardinality probability hypothesis density (CPHD) filter is run at each sensor node. Due to the fact that each sensor node has a limited FoV, the commonly adopted fusion methods become unreliable. In fact, the monitored area of multiple sensor nodes consists of several parts that are either exclusive of a single node, i.e. exclusive FoVs (eFoVs) or common to multiple (at least two) nodes, i.e. common FoVs (cFoVs). In this setting, the crucial issue is how to account for this different information sets in the fusion rule. The problem is particularly challenging when the knowledge of the FoVs is unreliable, for example because of the presence of obstacles and target misdetection, or when the FoVs are time-varying. Considering these issues, we propose an effective fusion algorithm for the case of unknown FoVs, where: i) the intensity function is decomposed into multiple sub-intensities/groups by means of a clustering algorithm; ii) the corresponding cardinality distribution is reconstructed by approximating the target random finite set (RFS) as multi-Bernoulli; and iii) fusion is performed in parallel according to either generalized covariance intersection (GCI) or arithmetic average (AA) rule. Simulation experiments are provided to demonstrate the effectiveness of the proposed approach.
\end{abstract}

Index Terms-CPHD, fields-of-view, clustering, Gaussian mixture, GCI fusion, AA fusion.

\section{INTRODUCTION}

$\mathbf{S}$ ENSOR networks have undergone a rapid growth thanks to the development of low-cost and low energyconsumption devices. One of the major tasks for such networks is distributed multi-target tracking (DMTT), wherein sensor nodes perform local MTT and fusion by exchanging multitarget information with the other nodes. So far, DMTT has been successfully adopted in many fields such as environmental monitoring, air/ground/maritime surveillance, and sensor management $[1]-[5]$.

Broadly speaking, the aim of MTT is the estimation of target number and states. A major problem challenge in achieving this objective is the unknown association of measurements to targets. In this respect, the recently developed random

This work was supported in part by the National Natural Science Foundation of China under Grant 61771110, in part by the Chang Jiang Scholars Program, in part by the 111 Project No. B17008.

G. Li, W. Yi, and L. Kong are with the School of Information and Communication Engineering, University of Electronic Science and Technology of China, Chengdu 611731, China, and G. Li is also with the Dipartimento di Ingegneria dell'Informazione, Università di Firenze, Florence 50139, Italy (e-mail: guchong.li@hotmail.com, kussoyi@gmail.com, lingjiang.kong@gmail.com).

G. Battistelli and L. Chisci are with the Dipartimento di Ingegneria dell'Informazione, Università di Firenze, Florence 50139, Italy (e-mail: giorgio.battistelli@unifi.it, luigi.chisci@unifi.it). finite set (RFS) approach [5], [6] has provided some effective solutions, such as the probability hypothesis density (PHD) [7] and cardinalized PHD (CPHD) [6] filters, in which the target RFS is modeled as Poisson or, respectively, independent identically distributed (IID) cluster process. Instead of propagating the first-order moment and cardinality distribution like PHD and CPHD filters, another filtering approach, named multi-Bernoulli (MB) filter [8], [9], propagates the multi-target posterior density. Some effective implementations for these filters can be found by resorting to either the Gaussian Mixture (GM) [8], [10], [11] or sequential Monte Carlo (SMC) [8], [12], [13] approaches.

Another key issue for DMTT is the selection of the fusion rule. Because of the unknown common information of sensor nodes, optimal fusion [14] is ruled out. At the moment, there are two commonly adopted approaches for fusing multiple RFS densities: generalized covariance intersection (GCI) $[15]-[18]$ and arithmetic average (AA) [19]-[22].

- GCI rule: The GCI rule, which provides the weighted geometrical average (GA) of the local RFS densities, is also named exponential mixture density (EMD) in some papers [15]. From an information-theoretic point of view, it can be derived according to the minimum information gain (MIG) criterion wherein the Kullback-Leibler divergence (KLD) [23] is regarded as distance between densities [16], [17], [24]. It has been mathematically proved that GCI fusion can effectively avoid double-counting of common information. Effective DMTT algorithms have been developed that combine GCI with several RFS-based (e.g., PHD, CPHD, multiBernoulli) filters, [15], [16], [25]-[27]. It should be noted, however, that the GCI rule suffers from lack of robustness against high misdetection rates [20].

- $A A$ rule: The AA rule provides a weighted arithmetic average (AA) of the local RFS densities, which also avoids the double-counting problem. From an information-theoretic viewpoint, AA fusion can be derived according to the minimum information loss (MIL) criterion based on KLD [20], [28]. In fact, while AA fusion of Poisson processes is no longer a Poisson process, the best fused Poisson process according to the MIL criterion can be obtained by arithmetically averaging the PHDs of the densities to be fused. Similarly, for IID cluster processes, the best fused IID cluster process according to the MIL criterion can be obtained by arithmetically averaging both cardinality distributions and PHDs. [20]. Compared to the GCI rule, the AA rule shows its benefits or potential to alleviate the misdetection [20], [29] and cardinality inconsistency [30] 
problems. DMTT algorithms combining RFS-based filters and AA fusion have been proposed in [20], [21], [29], [31].

Although much progress has been made in DMTT, a relevant, not yet completely addressed, issue is that sensor nodes have limited fields-of-view (FoVs), i.e. limited sensing range and/or angle. In such cases, how to extend the local FoVs to the overall FoV (union of all local FoVs) and achieve global tracking still remains a challenge. Unfortunately, the standard fusion rules (both GCI and AA) cannot satisfactorily deal with this objective. More specifically, GCI fusion tends to make targets outside the common FoV disappear because of its multiplicative (GA) nature, while AA fusion preserves such targets at the expense of decreasing their weights. Motivated by this inadequacy of the existing fusion rules, some work, briefly summarized hereafter, has been developed to handle multi-sensor fusion with multiple FoVs.

- Within the GCI rule framework, some simple but effective strategies, that consider the distance between Gaussian components (GCs) or particles to fuse nearby targets while keeping the targets that are in the exclusive FoV of only one node, are presented in [32]. Moreover, a solution to handle multiple FoVs in the context of the simultaneous localization and mapping (SLAM) is developed in [33]. Recently, a more principled method, where the overall state space is decomposed into sub-spaces, is proposed in [34]. Specifically, the method in [34] exploits parallelized GCI fusion in the common FoVs (cFoVs) and a compensation strategy in the exclusive FoVs (eFoVs). Moreover, the issue of multiple FoVs with GCI fusion has also been preliminarily investigated in the context of labeled RFS-based filters [35]-[37].

- Within the AA rule framework, a diffusion-based distributed SMC-PHD filter using sensors with limited sensing range has been presented in [38]. Recently, AA fusion for labeledRFS filters under multiple FoVs has been investigated in [29].

In general, for unlabeled RFS-based filters, the above mentioned approaches focus on the PHD filter. To the best of our knowledge, fusion of CPHDs under multiple different FoVs has not so far been addressed. In this paper, we tackle distributed CPHD-based filtering over a sensor network wherein nodes have different FoVs, and either GCI or AA fusion are employed in order to combine information among local CPHD filters. Summing up, the contributions of this work are the following.

1) We demonstrate the impracticality of fusion under known FoVs. Although exact knowledge of the FoVs could easily accomplish integration of the local FoVs into the overall FoV, it is shown that the unavoidable uncertainties due to, e.g., targets near the boundary of the FoV and misdetection of targets within the FoV caused by obstacles, can easily lead to overestimation or underestimation of the target number. This analysis motivates investigation of the more realistic case of fusion under unknown FoVs.

2) We propose a robust distributed CPHD-based filter with unknown FoVs. In this context, adopting a GM implementation, the intensity functions to be fused are decomposed into sub-intensity functions by clustering the GCs. Then, to re-construct a CPHD form for each sub-intensity, the cardinality distribution is constructed by approximating the target RFS as multi-Bernoulli. Next, the CPHDbased fusion between sub-intensities is carried out. After fusion, a new IID cluster process is constructed via convolution. The proposed solution is implemented using a GM approach.

3) We show that the proposed clustering-based fusion strategy can be interpreted in terms of decomposition of the state space, and provide an analysis of the related approximation error. Simulation experiments are also given to demonstrate the effectiveness of the proposed approach.

The outline of the rest of the paper is as follows. Section II introduces the background, and the problem statement is provided in Section III. The proposed fusion algorithm is described in Section IV. Simulation experiments are provided in Section V, and conclusions are drawn in Section VI.

\section{BACKGROUND}

\section{A. Sensor network}

Suppose that $N$ sensor nodes make up a sensor network wherein each node can communicate with its neighbors, sense the environment and locally process the available data. Mathematically speaking, the network is represented by a graph $(\mathcal{N}, \mathcal{A})$, where $\mathcal{N}=\{1,2, \cdots, N\}$ denotes the set of nodes and $\mathcal{A} \subseteq \mathcal{N} \times \mathcal{N}$ the set of arcs (links). For any two nodes $i$ and $j$, there exists arc $(i, j) \in \mathcal{A}$ if node $j$ can receive data from node $i$. Moreover, the in-neighborood of node $i$ is denoted as $\mathcal{N}^{i}=\{j \in \mathcal{N}:(j, i) \in \mathcal{A}\}$. The local FoV of node $l \in \mathcal{N}$, which represents the region wherein it can detect targets, is denoted as $\mathcal{S}_{l}$. Accordingly, $\mathcal{S}=\cup_{l=1}^{N} \mathcal{S}^{l}$ is the overall FoV defined as the union of all local FoVs. Further, the $\mathrm{cFoV}$ between nodes $i$ and $j$ is defined as $\digamma_{c}^{i, j}=\mathcal{S}^{i} \cap \mathcal{S}^{j}$ and the eFoVs for nodes $i$ and $j$ are, respectively, denoted as $\digamma_{e}^{i, j}=\mathcal{S}^{i} \backslash \digamma_{c}^{i, j}$ and $\digamma_{e}^{j, i}=\mathcal{S}^{j} \backslash \digamma_{c}^{i, j}$.

\section{B. Multi-target Bayesian filter}

In random set tracking, targets and measurements, at time $k$, are regarded as RFSs

$$
\begin{aligned}
X_{k} & =\left\{x_{k}^{1}, \cdots, x_{k}^{n_{k}}\right\} \in \mathcal{F}(\mathbb{X}), \\
Z_{k} & =\left\{z_{k}^{1}, \cdots, z_{k}^{m_{k}}\right\} \in \mathcal{F}(\mathbb{Z}),
\end{aligned}
$$

where: $n_{k}$ and $m_{k}$ denote the unknown number of targets and, respectively, known number of measurements at time $k ; \mathbb{X}$ and $\mathbb{Z}$ denote the state and, respectively, measurement space; $x_{k}^{i} \in \mathbb{X}$ and $z_{k}^{j} \in \mathbb{Z}$ are the $i$-th target state and, respectively, $j$-th measurement at time $k ; \mathcal{F}(\mathbb{X})$ denotes the set of finite subsets of $\mathbb{X}$.

Given the multi-target posterior density $f_{k-1 \mid k-1}(\cdot)$ at time $k-1$ and measurement set $Z_{k}$ at time $k$, the multi-target Bayes 
recursion can be expressed as follows

$$
\begin{aligned}
f_{k \mid k-1}\left(X_{k}\right) & =\int \varphi_{k \mid k-1}\left(X_{k} \mid X\right) f_{k-1 \mid k-1}(X) \delta X, \\
f_{k \mid k}\left(X_{k}\right) & =\frac{\ell_{k}\left(Z_{k} \mid X_{k}\right) f_{k \mid k-1}\left(X_{k}\right)}{\int \ell_{k}\left(Z_{k} \mid X\right) f_{k \mid k-1}(X) \delta X},
\end{aligned}
$$

where $\varphi_{k \mid k-1}(\cdot \mid \cdot)$ and $\ell_{k}(\cdot \mid \cdot)$ are the multi-target transition density describing the time evolution of the multi-target state and, respectively, the multi-object likelihood describing the multi-target measurement model, respectively. The integrals in (1]) and 22] are intended in the set integral sense [6].

In general, the multi-target Bayesian filter can be hardly implemented unless the multi-target set $X_{k}$ is approximated, at each time $k$, by some special RFS. Hereafter, some typically used RFS approximations are summarized.

- Multi-target Poisson RFS: the multi-target posterior takes the form

$$
f_{k \mid k}\left(X_{k}\right)=e^{-\mu_{k \mid k}} \prod_{i=1}^{n} \mu_{k \mid k} s_{k \mid k}\left(x_{k}^{i}\right),
$$

where $\mu_{k \mid k}$ denotes the expected number of targets and $s_{k \mid k}$ is the target location density. Notice that all the information contained in a Poisson RFS can be summarized by its PHD (also called intensity function)

$$
v_{k \mid k}(x)=\mu_{k \mid k} s_{k \mid k}(x),
$$

which represents the first moment of the multi-target density. The integral of the PHD $v_{k \mid k}$ in any region of the state space corresponds to the expected number of targets contained in that region.

- IID cluster process: the multi-target posterior takes the form

$$
f_{k \mid k}\left(X_{k}\right)=n ! p_{k \mid k}(n) \prod_{i=1}^{n} s_{k \mid k}\left(x_{k}^{i}\right),
$$

where $p_{k \mid k}$ is the cardinality distribution and $s_{k \mid k}$ is again the target location density. The information contained in an IID cluster process can be summarized by the pair $\left(p_{k \mid k}, s_{k \mid k}\right)$ or, equivalently, by the pair $\left(p_{k \mid k}, v_{k \mid k}\right)$ where the PHD is as in (4) with

$$
\mu_{k \mid k}=\sum_{n=0}^{\infty} n p_{k \mid k}(n)
$$

- Multi-Bernoulli RFS: the multi-target posterior takes the form

$$
\begin{aligned}
f_{k \mid k}\left(X_{k}\right) & =n ! \prod_{i=1}^{n}\left(1-r_{i}\right) \\
& \times \sum_{1 \leq i_{1} \neq \cdots \neq i_{n} \leq M} \prod_{j=1}^{n} \frac{r_{i_{j}} s_{i_{j}}\left(x_{j}\right)}{1-r_{i_{j}}},
\end{aligned}
$$

where $M$ is the number of Bernoulli components, $r_{i}$ and $s_{i}$ are the probability of existence and, respectively, location density of the $i$-th Bernoulli component.

\section{Fusion rule}

Given multiple multi-target posterior densities, $\left\{\omega^{i}, f^{i}(X)\right\}$ with $\sum_{i} \omega^{i}=1$, the GCI and AA fusion rules will be consid- ered. In particular, assuming that the densities to be fused take the form of IID cluster processes $f^{i}(X)=n ! p^{i}(n) \prod_{x \in X} s^{i}(x)$, the GCI fusion provides

$$
\begin{aligned}
& \bar{s}_{\mathrm{GCI}}(x)=\frac{\prod_{i}\left[s^{i}(x)\right]^{\omega^{i}}}{\int \prod_{i}\left[s^{i}(x)\right]^{\omega^{i}} d x}, \\
& \bar{p}_{\mathrm{GCI}}(n)=\frac{\prod_{i}\left[p^{i}(n)\right]^{\omega^{i}}\left\{\int \prod_{i}\left[s^{i}(x)\right]^{\omega^{i}} d x\right\}^{n}}{\sum_{m=0}^{\infty} \prod_{i}\left[p^{i}(m)\right]^{\omega^{i}}\left\{\int \prod_{i}\left[s^{i}(x)\right]^{\omega^{i}} d x\right\}^{m}} .
\end{aligned}
$$

The AA fusion instead yields

$$
\begin{aligned}
& \bar{v}_{\mathrm{AA}}(x)=\sum_{i} \omega^{i} v^{i}(x), \\
& \bar{p}_{\mathrm{AA}}(n)=\sum_{i} \omega^{i} p^{i}(n),
\end{aligned}
$$

or, equivalently, in terms of target location density

$$
\bar{s}_{\mathrm{AA}}(x)=\frac{1}{\sum_{n=0} n \bar{p}_{\mathrm{AA}}(n)} \sum_{i}\left(\sum_{n=0} n p^{i}(n)\right) \omega^{i} s^{i}(x) .
$$

There are essentially two ways to implement CPHD-based fusion, namely the GM approach [16], [20] and, respectively, SMC (particle) approach [15].

\section{Problem Statement}

Although the GCI rule has been widely applied in DMTT scenarios, most of the papers assume that all nodes share the same FoV [16], [40], [41]. In practice, however, different nodes turn out to have different FoVs. As a matter of fact, each sensor node can only detect targets over a portion of the area of interest and then exchanges information with the linked nodes (neighbors) aiming to extend the monitored area as much as possible, hopefully to the union of the FoVs of all sensor nodes. In this respect, fusion has to be handled with care, otherwise it could be counterproductive. For instance, because of its multiplicative nature [see [8] ], a direct application of the GCI fusion rule would tend to make targets outside the common FoV disappear. Further, using AA fusion instead of GCI would only partially solve the problem since AA fusion tends to preserve all targets but with a reduced weight. In fact, the estimated number of targets after fusion corresponds to the AA of the estimated number of targets of the local node, thus resulting in a severe underestimation when the FoVs overlap only partially.

To sum up, the fusion with multiple FoVs is still a thorny issue. Although PHD-based fusion with different FoVs has already been investigated [34], [38] it is worth to point out that the PHD approach to DMTT, only keeping first-order moment information, may not be able to provide the desired tracking performance. In contrast, the CPHD approach might yield considerable performance improvements thus motivating our interest in this paper for the unaddressed topic of CPHDbased fusion with different FoVs. 
To make the fusion robust to multiple FoVs, based on our previous work in [34], we resort to the idea of partitioning the global state space $\mathbb{X}$ into $G$ disjoint subspaces, i.e.,

$$
\begin{aligned}
& \mathbb{X}=\mathbb{X}_{1} \cup \mathbb{X}_{2} \cup \cdots \cup \mathbb{X}_{G}, \\
& \mathbb{X}_{g} \cap \mathbb{X}_{g^{\prime}}=\emptyset, \quad \forall g \neq g^{\prime} .
\end{aligned}
$$

Accordingly, the target set $X_{k}$ is partitioned as the union of $G$ disjoint RFSs

$$
X_{k}=X_{k, 1} \cup X_{k, 2} \cup \cdots \cup X_{k, G},
$$

with $X_{k, g}=X_{k} \cap \mathbb{X}_{g}$ representing the targets inside the subspace $\mathbb{X}_{g}$ at time $k$. For each $g$, we determine the set $\mathcal{N}_{k, g} \subseteq \mathcal{N}$ of the nodes of the sensor network having information on the target subset $X_{k, g}$ at time $k$. Then the idea is that for, each $g$, only the nodes belonging to $\mathcal{N}_{k, g}$ should be involved in the fusion because they are the only ones providing information.

\section{A. Distributed CPHD filtering with different FoVs}

Suppose now that each sensor node is running locally a CPHD filter so that, after the local correction step, the posterior density of each node $i$ takes the form of an IID cluster process with cardinality distribution $p_{k \mid k}^{i}$ and target location density $s_{k \mid k}^{i}$. Notice that, in order to simplify the notations, hereafter we will drop the time dependence and simply write $p^{i}$ and $s^{i}$ where it is understood that all quantities refer to the current time instant $k$. The key steps in applying the above-described idea to the fusion of IID cluster processes are:

a) for each node $i$, split the original IID cluster density $\left(p^{i}, s^{i}\right)$ into suitable sub-IID cluster densities $\left(p_{g}^{i}, s_{g}^{i}\right)$ each one corresponding to one of the subspaces $\mathbb{X}_{g}$;

b) for any $g$, carry out fusion between the sub-IID cluster densities $\left(p_{g}^{i}, s_{g}^{i}\right)$ with $i \in \mathcal{N}_{k, g}$ to get the fused sub-IID cluster density $\left(\bar{p}_{g}, \bar{s}_{g}\right)$

c) after fusion, combine the fused sub-IID cluster density $\left(\bar{p}_{g}, \bar{s}_{g}\right)$ with $g=1, \ldots, G$ to get the total fused IID cluster density $(\bar{p}, \bar{s})$.

Notice that in the above procedure, each target subset $X_{k, g}$ is modelled as an IID cluster process. Accordingly, the total target set $X_{k}$ is modelled as a superposition (independent union) of IID cluster processes. This means that, given the fused cardinality distributions $\bar{p}_{g}$ and PHD $\bar{v}_{g}$ pertaining to each subset $X_{k, g}$, the total fused cardinality distribution $\bar{p}$ and PHD $\bar{v}$ in step c) can be readily obtained as

$$
\begin{aligned}
& \bar{p}(n)=\left(\bar{p}_{1} * \cdots * \bar{p}_{G}\right)(n), \\
& \bar{v}(x)=\bar{v}_{1}(x)+\ldots+\bar{v}_{n}(x),
\end{aligned}
$$

where $*$ stands for discrete convolution.

The converse operation, that is the splitting of the total IID cluster density $\left(p^{i}, s^{i}\right)$ into the sub-IID cluster densities $\left(p_{g}^{i}, s_{g}^{i}\right)$ which has to be performed in step a), can be carried out by resorting to a multi-Bernoulli approximation as proposed in [5, section 9.4.2].

First of all, notice that the total intensity $v^{i}$ can be readily splitted as

$$
v^{i}(x)=v_{1}^{i}(x)+\ldots+v_{G}^{i}(x),
$$

where each sub-intensity is obtained as

$$
v_{g}^{i}(x)=1_{\mathbb{X}_{g}}(x) v^{i}(x),
$$

$1_{\mathbb{X}_{g}}(x)$ denoting the indicator function of set $\mathbb{X}_{g}$.

Let us assume now, without loss of generality, that each sub-intensity can be expressed as

$$
v_{g}^{i}(x)=\sum_{q=1}^{J_{g}^{i}} \alpha_{g, q}^{i} f_{g, q}^{i}(x),
$$

where $\alpha_{g, q}^{i} \in(0,1)$ and $\int_{\mathbb{X}} f_{g, q}^{i}(x) d x=1$. In this way, the existence probabilities $\alpha_{g, q}^{i}$ and spatial probability density functions (PDFs) $f_{g, q}^{i}$ provide the best multi-Bernoulli approximation. Then, exploiting [5, eqn. (9.29)], the cardinality distribution of the $g$-th sub-IID cluster process can be computed as follows

$$
p_{g}^{i}(n)=\prod_{j=1}^{J_{g}^{i}}\left(1-\alpha_{g, q}^{i}\right) \sigma_{J_{g}^{i}, n}\left(\frac{\alpha_{g, 1}^{i}}{1-\alpha_{g, 1}^{i}}, \cdots, \frac{\alpha_{g, J_{g}^{i}}^{i}}{1-\alpha_{g, J_{g}^{i}}^{i}}\right)
$$

where

$\sigma_{m, n}\left(\beta_{1}, \cdots, \beta_{m}\right)=\left\{\begin{array}{cl}1 & \text { if } n=0 \\ \sum_{1 \leq i_{1}<\cdots<i_{n} \leq m} \prod_{j=1}^{n} \beta_{i_{j}} & \text { if } 1 \leq n \leq m \\ 0 & \text { if } i>m\end{array}\right.$

is called elementary symmetric function (ESF) of degree $n$ in $m$ variables.

\section{B. On the decomposition of the state space}

The fusion procedure described in the previous section presupposes that the state space is already partitioned into disjoints sets $\mathbb{X}_{1}, \ldots, \mathbb{X}_{G}$ and that, for each $\mathbb{X}_{g}$, we know which of the sensor nodes have information on the targets belonging to $\mathbb{X}_{g}$ (i.e., for each $g$ the set $\mathcal{N}_{k, g}$ is supposed to be known).

If the FoVs were precisely known, a possible common-sense strategy could be to define the state space partition on the basis of the FoVs so as to fuse information within the $\mathrm{cFoV}$ while copying information from the eFoVs. Unfortunately, such common-sense fusion strategy lacks of robustness with respect to the unavoidable uncertainties on the FoVs as it can be seen from the two examples illustrated in Fig. 1, concerning fusion between two nodes.

- Targets located in the eFoV.

In the example of Fig. 1 1 a), the local density $f^{i}(\cdot)$ of node $i$ has a Gaussian Component (GC) located in the eFoV $\digamma_{e}^{i, j}$ and similarly for node $j$ in $\digamma_{e}^{j, i}$. Due to their closeness, the two GCs clearly correspond to the same target. Then, applying common-sense fusion, they are both copied into the fused density thus implying an overestimation of the target number.

- Targets located in the cFoV.

In the example of Fig. 1 (b), the local density $f^{i}(\cdot)$ of node $i$ has a single GC located in the $\mathrm{cFoV} \digamma_{c}^{i, j}$ while for node $j$ there are two GCs in $\digamma_{c}^{i, j}$. Notice that two GCs correspond 


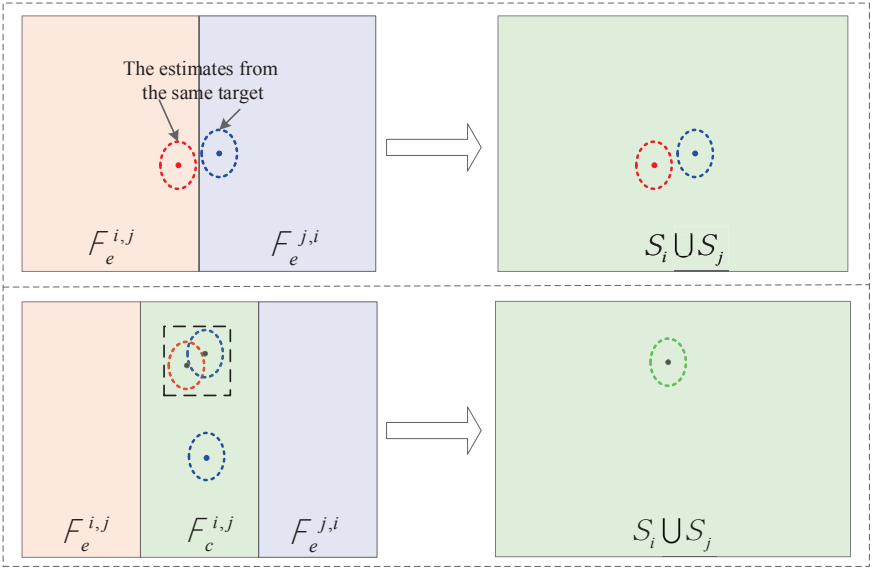

Fig. 1. (a) Two GCs, actually representing the same target, are located in the eFoVs of the two fusing nodes $i$ and $j$. Hence, they are both copied in the fused density thus implying overestimation of the target number. (b) Three GCs are located in the cFoV of nodes $i$ and $j$; two of them, corresponding to the same target, are merged after fusion while the third one, corresponding to a target detected by node $j$ but node by node $i$, disappears after fusion thus implying underestimaton of the target number.

to the same target and, after fusion, are merged into a single one while the other GC, which corresponds to a target detected by node $j$ but undetected by node $i$, tends to be removed after fusion. In this case, underestimation of the target number occurs.

Further, the common-sense startegy cannot be applied when the FoVs are not perfectly known (for example due to occlusions) or when the sensors have information also outside their current FoVs (for example because the FoVs are time-varying or because of the fusion feedback at previous time instants).

For the above reasons, in this paper we give up on defining the state-space partition on the basis of the sensor FoVs and, instead, propose a procedure that exploit clustering to directly split the original IID cluster density into suitable sub-IID cluster densities and, then, carry out fusion without knowledge of the FoVs.

\section{ROBUST DISTRIBUTED CPHD FILTERING WITH MULTIPLE FOVS}

To make the CPHD-based fusion immune to multiple FoVs, a robust fusion strategy based on clustering is now proposed. Section IV-A provides a detailed description of the fusion algorithm, while Section IV-B shows how the proposed approach is related to the decomposition of the state space introduced in Section II. Without loss of generality, pairwise fusion between two nodes, say 1 and $2 \in \mathcal{N}^{1}$, will be considered but the resulting fusion approach will admit a straightforward extension to an arbitrary number of fusing nodes, i.e., it can be applied simultaneously to any subset of $\mathcal{N}$.

\section{A. Robust CPHD-based fusion with unknown FoVs}

Following a GM implementation, it is assumed that the local intensities of the sensor nodes are expressed as

$$
v^{i}(x)=\sum_{p=1}^{J^{i}} \alpha_{p}^{i} \mathcal{G}\left(x ; m_{p}^{i}, P_{p}^{i}\right)
$$

for $i \in\{1,2\}$, where $\alpha_{p}^{i} \in(0,1)$ and $\mathcal{G}(x ; m, P)$ denotes a Gaussian PDF with mean $m$ and covariance $P$. The proposed fusion algorithm consists of four steps detailed hereafter.

1) Clustering of GCs: In order to determine whether two generic GCs need to be grouped into the same cluster or not, the idea is to compare a suitable distance between the GCs to an appropriately chosen threshold. Specifically, let $\mathcal{G}_{p}^{i}$ and $\mathcal{G}_{q}^{j}$ be two generic GCs 1 with $i, j \in\{1,2\}$. Then, their distance is defined in terms of the corrected Mahalanobis distance,

$$
d\left(\mathcal{G}_{p}^{i}, \mathcal{G}_{q}^{j}\right)=\left(m_{p}^{i}-m_{q}^{j}\right)^{\top}\left(P_{p}^{i}+P_{q}^{j}\right)^{-1}\left(m_{p}^{i}-m_{q}^{j}\right),
$$

If $d\left(\mathcal{G}_{p}^{i}, \mathcal{G}_{q}^{j}\right)$ is smaller than a preset gating threshold $\rho$, then $\mathcal{G}_{p}^{i}$ and $\mathcal{G}_{q}^{j}$ are assigned to the same cluster. It is clear that the farther apart the two GCs are, the less similar they are. As a matter of fact, a large distance makes the common information between GCs negligible. As a consequence, the intensity function can be approximately split into sub-intensity functions corresponding to the obtained clusters. The detailed clustering process is provided in Algorithm 1.

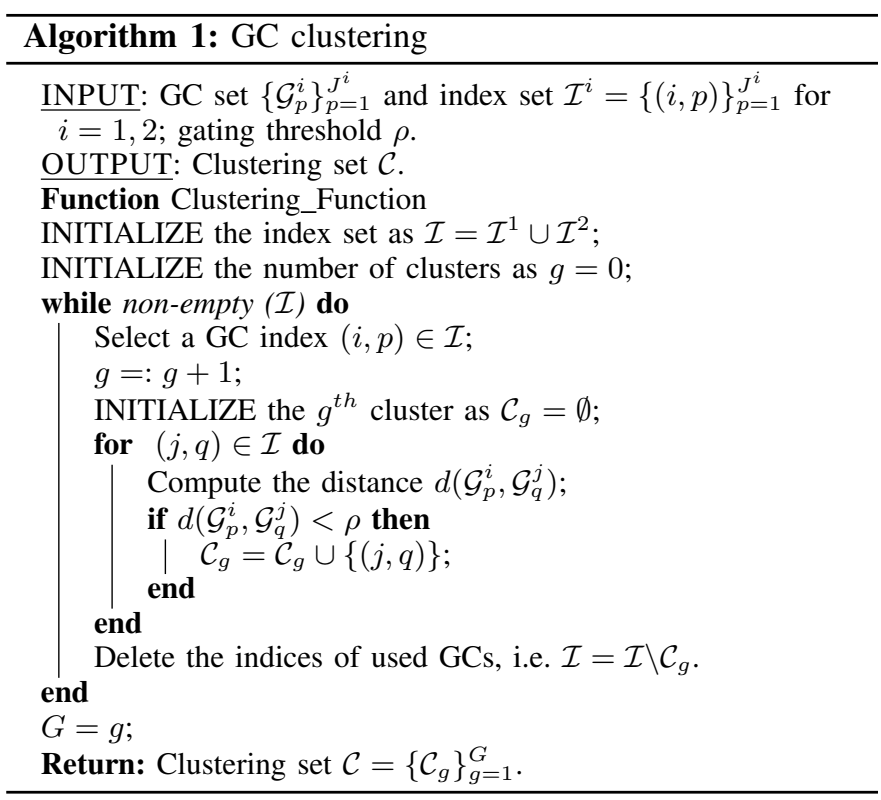

Based on the clustering process, a feasible definition of the clustering set is given as follows.

Definition 1. A set $\mathcal{C}=\left\{\mathcal{C}_{1}, \cdots, \mathcal{C}_{G}\right\}$ with $G$ clusters of $G C$ indices is a clustering set if the following two conditions hold:

for any cluster $\mathcal{C}_{g}$ and for any $(i, p) \in \mathcal{C}_{g}$, there exists $(j, q) \in \mathcal{C}_{g}$ such that

$$
d\left(\mathcal{G}_{p}^{i}, \mathcal{G}_{q}^{j}\right) \leq \rho
$$

for any $(i, p) \in \mathcal{C}_{g}$ and $(j, q) \in \mathcal{C}_{g^{\prime}}$ with $g \neq g^{\prime}$ then

$$
d\left(\mathcal{G}_{p}^{i}, \mathcal{G}_{q}^{j}\right)>\rho
$$

where $\rho$ is the predefined gating threshold.

Note that 23 is automatically satisfied according to the distance thresholding of Algorithm 1, while 24 may not be

\footnotetext{
${ }^{1}$ For convenience, the GC, $\mathcal{G}\left(x ; m_{p}^{i}, P_{p}^{i}\right)$, is simply denoted as $\mathcal{G}_{p}^{i}$, and similarly for $\mathcal{G}_{q}^{j}$.
} 
satisfied. In order to ensure also (24), we resort to the so-called union-find set (UFS) algorithm so that the distance between any two GCs from different clusters is larger than $\rho$, meaning that the clusters are well-separated.

As a result of clustering, the intensities of nodes 1 and 2 can be decomposed as

$$
v^{i}(x)=\sum_{g=1}^{G} \hat{v}_{g}^{i}(x)
$$

for $i \in\{1,2\}$ where each sub-intensity $\hat{v}_{g}^{i}(x)$ contains the GCs whose index $(i, p)$ belongs to $\mathcal{C}_{g}$. In particular, let $J_{g}^{i}$ denote the number of GCs of node $i$ belonging to cluster $\mathcal{C}_{g}$. Then, when $J_{g}^{i}>0$ the corresponding sub-intensity can be represented as

$$
\hat{v}_{g}^{i}(x)=\sum_{p=1}^{J_{g}^{i}} \alpha_{g, p}^{i} \mathcal{G}\left(x ; m_{g, p}^{i}, P_{g, p}^{i}\right) .
$$

Conversely, when $J_{g}^{i}=0$ we simply have $\hat{v}_{g}^{i}(x)=0$. This latter case corresponds to the situation in which node $i$ does not have any GC in cluster $\mathcal{C}_{g}$. In the proposed fusion strategy, the sub-intensities $\hat{v}_{g}^{i}$ obtained through clustering are used in place of the sub-intensities $v_{g}^{i}$ obtained according to the partitioning of the state space as in (17). Then, the set $\mathcal{N}_{k, g}$ is defined as $\mathcal{N}_{k, g}=\left\{i: J_{g}^{i}>0\right\}$ so that, for each cluster $\mathcal{C}_{g}$, only the sensor nodes having GCs belonging to such a cluster are involved in the fusion.

Remark 1. It is clear that clustering highly depends on the selection of the threshold $\rho$.

- Large $\rho$ might lead to group GCs from different targets into the same cluster. Consider two GCs from node $1, \mathcal{G}_{1}^{1} \in \digamma_{c}^{1,2}$ and $\mathcal{G}_{2}^{1} \in \digamma_{e}^{1,2}$, and one $G C$ from node $2, \mathcal{G}_{1}^{2} \in \digamma_{c}^{1,2}$, where $\mathcal{G}_{1}^{1}$ and $\mathcal{G}_{1}^{2}$ are from the same target. If the three $G C s$ are grouped into only one cluster, then the target represented by $\mathcal{G}_{2}^{2}$ will not be matched by any $G C$ of node 1 so that the number of targets will be underestimated after fusion.

- Small $\rho$ may enforce the GCs from the same target to be grouped into different clusters. For instance, if $v^{1}(x)=$ $0.9 \mathcal{G}_{1}^{1} \in \digamma_{c}^{1,2}$ and $v^{2}(x)=\mathcal{G}_{1}^{2} \in \digamma_{c}^{1,2}$ with $d\left(\mathcal{G}_{1}^{1}, \mathcal{G}_{1}^{2}\right)>\rho$, it can happen that these GCs are grouped into two different clusters even if they correspond to the same target. In such a case, the number of targets will be overestimated.

Hence, the selection of $\rho$ is a trade-off between underestimation and overestimation. For scenarios with well-separated targets, $\rho$ can be easily chosen. A more formal analysis of the role played by the gating threshold $\rho$ will be given in Section $I V-B$.

2) Construction of the cardinality distribution of each cluster: For the CPHD filter, one major advantage is the use of the cardinality distribution to estimate the number of targets according to the maximum a posteriori probability (MAP) criterion rather than the expected a posteriori (EAP) criterion adopted by the PHD filter. Hence, the construction of the cardinality distribution is needed.

For the obtained $g$-th cluster and the corresponding subintensities $\hat{v}_{g}^{i}(x), i \in\{1,2\}$, by using the Bernoulli ap- proximation, the cardinality distributions can be obtained as follows:

$$
\begin{aligned}
& \hat{p}_{g}^{i}(n) \\
& =\left(\prod_{p=1}^{J_{g}^{i}}\left(1-\alpha_{g, p}^{i}\right)\right) \sigma_{J_{g}^{i}, n}\left(\frac{\alpha_{g, 1}^{i}}{1-\alpha_{g, 1}^{i}}, \cdots, \frac{\alpha_{g, J_{g}^{i}}^{i}}{1-\alpha_{g, J_{g}^{i}}^{i}}\right)
\end{aligned}
$$

for $i \in\{1,2\}$.

3) Fusion for each cluster: For any cluster $\mathcal{C}_{g}, g \in$ $\{1, \cdots, G\}$, there are two possible cases depending on the origin of the GCs within the cluster.

- all GCs belong to a single node $i$ (i.e. $\mathcal{N}_{k, g}=\{i\}$ ): keep the sub-intensity and the cardinality distribution of such a node

$$
\begin{aligned}
& \bar{v}_{g}(x)=\hat{v}_{g}^{i}(x), \\
& \bar{p}_{g}(x)=\hat{p}_{g}^{i}(x) .
\end{aligned}
$$

- GCs belong to both nodes 1 and 2 (i.e. $\mathcal{N}_{k, g}=\{1,2\}$ ): $\left(\hat{v}_{g}^{1}, \hat{p}_{g}^{1}\right)$ and $\left(\hat{v}_{g}^{2}, \hat{p}_{g}^{2}\right)$ need to be fused by using either the GCI or the AA fusion rule.

GCI fusion The fused target location density for cluster $\mathcal{C}_{g}$ can be obtained by resorting to any of the available techniques for computing the GA of GMs. For example, when the approximation of [16] is adopted, the fused target location density takes the form

$$
\bar{s}_{g}(x)=C^{-1} \sum_{p=1}^{J_{g}^{1}} \sum_{q=1}^{J_{g}^{2}} \alpha_{p q}^{1,2} \mathcal{G}\left(x ; m_{p q}^{1,2}, P_{p q}^{1,2}\right)
$$

where

$$
\begin{aligned}
P_{p q}^{1,2}= & {\left[\omega\left(P_{g, p}^{1}\right)^{-1}+(1-\omega)\left(P_{g, q}^{2}\right)^{-1}\right]^{-1}, } \\
m_{p q}^{1,2}= & P_{p q}^{1,2}\left[\omega\left(P_{g, p}^{1}\right)^{-1} m_{g, p}^{1}+(1-\omega)\left(P_{g, q}^{2}\right)^{-1} m_{g, q}^{2}\right], \\
\alpha_{p q}^{1,2}= & \left(\alpha_{g, p}^{1}\right)^{\omega}\left(\alpha_{g, q}^{2}\right)^{1-\omega} \kappa\left(\omega, P_{g, p}^{1}\right) \kappa\left(1-\omega, P_{g, q}^{2}\right) \\
& \cdot \mathcal{G}\left(m_{g, p}^{1}-m_{g, q}^{2} ; 0, \frac{P_{g, p}^{1}}{\omega}+\frac{P_{g, q}^{2}}{1-\omega}\right)
\end{aligned}
$$

and

$$
\begin{gathered}
C=\sum_{p=1}^{J_{g}^{1}} \sum_{q=1}^{J_{g}^{2}} \alpha_{p q}^{1,2}, \\
\kappa(\omega, P)=\frac{\left[\operatorname{det}\left(2 \pi P \omega^{-1}\right)\right]^{\frac{1}{2}}}{[\operatorname{det}(2 \pi P)]^{\frac{\omega}{2}}} .
\end{gathered}
$$

Substituting 30 into 9 , the cardinality distribution $\bar{p}_{g}(n)$ can be obtained. Moreover, the fused sub-intensity function can be expressed as follows:

$$
\bar{v}_{g}(x)=\bar{s}_{g}(x) \sum_{n \geq 0} n \bar{p}_{g}(n) .
$$

AA fusion The fused sub-intensity function and cardinality distribution can be readily computed without any approxi- 
mation as follows

$$
\begin{aligned}
\bar{v}_{g}(x)= & \omega \sum_{p=1}^{J_{g}^{1}} \alpha_{g, p}^{1} \mathcal{G}\left(x ; m_{g, p}^{1}, P_{g, p}^{1}\right) \\
& +(1-\omega) \sum_{q=0}^{J_{g}^{2}} \alpha_{g, q}^{2} \mathcal{G}\left(x ; m_{g, q}^{2}, P_{g, q}^{2}\right), \\
\bar{p}_{g}(n)= & \omega \hat{p}_{g}^{1}(n)+(1-\omega) \hat{p}_{g}^{2}(n) .
\end{aligned}
$$

4) Merging sub-IID cluster processes: Once the clusterwise fusion has been carried out, all fused sub-IID cluster processes should be merged into a single IID cluster process. The merging is obtained by summing all sub-intensity functions and convolving the corresponding cardinality distributions as follows:

$$
\begin{aligned}
\bar{v}(x) & =\sum_{g=1}^{G} \bar{v}_{g}(x), \\
\bar{p}^{i}(n) & =\left(\bar{p}_{1} * \bar{p}_{2} * \cdots * \bar{p}_{G}\right)(n) \\
& =\sum_{n_{1}+n_{2}+\cdots+n_{G}=n} \bar{p}_{1}\left(n_{1}\right) \cdot \bar{p}_{2}\left(n_{2}\right) \cdots \bar{p}_{G}\left(n_{G}\right) .
\end{aligned}
$$

Remark 2. It is worth to point out that clustering does not involve the weights of the GCs. That is to say, the focus is only on the spatial positions of targets but not on the existence probabilities. The following two cases need to be emphasized.

- If one target travels only in the FoV of one of the two fusing nodes, no fusion is performed and the GCs of the target will be preserved so that the target can be detected by both nodes. In such a case, both AA and GCI fusion will provide good cardinality estimation.

- Under low detection probability, it may happen that in the $c F o V$ there are two GCs from different nodes in the same cluster with comparatively different weights, i.e. one very small and the other greater than 0.5. In such a case, the target estimation accuracy of the fused GC, no matter whether GCI or AA fusion is adopted, can be improved but the weight of the fused GC may decrease compared to the initial GC with larger weight. Hence, cardinality may be underestimated. It should be noticed that in a sensor network, it is difficult to guarantee that all nodes can simultaneously detect all the targets. In this respect, GCI fusion, due to its multiplicative nature, is more sensitive to target misdetection than AA fusion which, on the other hand, has additive nature.

Remark 3. Although the above algorithmic description concerns only two sensor nodes (pairwise fusion), it is easy to extend it to an arbitrary number of nodes, e.g., by means of a sequence of pairwise fusions.

Remark 4. In alternative to the previously considered GM implementation, also a SMC (particle) approach, using deltafunction mixtures instead of GMs, could be adopted. In such a case, the Euclidean distance can be exploited in order to measure the closeness between particles in the clustering process. Further, the Kernel Density Estimation (KDE) method [44] could be used for GCI fusion of delta-function mixtures.
Remark 5. Notice that the proposed clustering-based fusion strategy is well-suited to being applied also in the context of distributed PHD filtering with different FoVs. In this case, clearly, the operations related to splitting and fusion of the cardinality distribution need not be performed.

\section{B. Approximation error analysis}

In this subsection, we provide an interpretation of the proposed clustering-based fusion strategy in terms of decomposition of the state space, and analyze the error induced by approximating the sub-intensities $v_{g}^{i}(x)$ with the GMs $\hat{v}_{g}^{i}(x)$.

To this end, given a GC $\mathcal{G}_{p}^{i}$ and a scalar $\delta$, let us consider the confidence ellipsoid

$$
\mathcal{E}_{p}^{i}(\delta)=\left\{x \in \mathbb{X}:\left(x-m_{p}^{i}\right)^{\top}\left(P_{p}^{i}\right)^{-1}\left(x-m_{p}\right) \leq \delta\right\} .
$$

Recall that, after step 1) of the proposed strategy, the index partition $\mathcal{C}=\left\{\mathcal{C}_{1}, \ldots, \mathcal{C}_{g}\right\}$ defines a clustering set satisfying both properties in Definition 1. As a consequence the following result holds.

Lemma 1. Let $\delta$ be such that $\delta \leq \rho / 4$, $\rho$ being the gating threshold. Then, for any $(i, p) \in \mathcal{C}_{g}$ and $(j, q) \in \mathcal{C}_{g^{\prime}}$ with $g \neq g^{\prime}$, we have

$$
\mathcal{E}_{p}^{i}(\delta) \cap \mathcal{E}_{q}^{j}(\delta)=\emptyset .
$$

Proof: see Appendix A.

In turn, Lemma 1 implies that we can partition the state space $\mathbb{X}$ into $G$ disjoint sets $\mathbb{X}_{1}, \ldots, \mathbb{X}_{G}$ having the properties that

$$
\begin{aligned}
& (i, p) \in \mathcal{C}_{g} \Longrightarrow \mathcal{E}_{p}^{i}(\delta) \subseteq \mathbb{X}_{g}, \\
& (i, p) \notin \mathcal{C}_{g} \Longrightarrow \mathcal{E}_{p}^{i}(\delta) \cap \mathbb{X}_{g}=\emptyset .
\end{aligned}
$$

Hence the clustering procedure based on the corrected Mahalanobis distance 22 implicitly defines a partitioning of the state space.

Consider now the sub-intensities $v_{g}^{i}(x)$ determined by splitting each $v^{i}(x)$ with respect to the above-defined partition

$$
v_{g}^{i}(x)=1_{\mathbb{X}_{g}}(x) v^{i}(x)=\sum_{p=1}^{J^{i}} \alpha_{p}^{i} 1_{\mathbb{X}_{g}}(x) \mathcal{G}_{p}^{i}(x),
$$

and the corresponding sub-intensities $\hat{v}_{g}^{i}(x)$ obtained by means of the proposed clustering procedure

$$
\hat{v}_{g}^{i}(x)=\sum_{p:(i, p) \in \mathcal{C}_{g}} \alpha_{p}^{i} \mathcal{G}_{p}^{i}(x) .
$$

Notice that the discrepancy between each pair of subintensities can be written as

$$
\begin{aligned}
v_{g}^{i}(x)-\hat{v}_{g}^{i}(x)= & \sum_{p:(i, p) \in \mathcal{C}_{g}} \alpha_{p}^{i}\left[1-1_{\mathbb{X}_{g}}(x)\right] \mathcal{G}_{p}^{i}(x) \\
& +\sum_{p:(i, p) \notin \mathcal{C}_{g}} \alpha_{p}^{i} 1_{\mathbb{X}_{g}}(x) \mathcal{G}_{p}^{i}(x) .
\end{aligned}
$$

Recall now that for a Gaussian PDF $\mathcal{G}_{p}^{i}$ we have

$$
\int_{\mathcal{E}_{p}^{i}(\delta)} \mathcal{G}_{p}^{i}(x) d x=F(\delta, \operatorname{dim}(x)),
$$


where $F(\delta, d)$ denotes the cumulative distribution function of the $\chi^{2}$-distribution with $d$ degrees of freedom. Then, by exploiting properties (40)- 41 , a bound on the approximation error (44) in $L_{1}$-norm can be obtained.

Theorem 1. Let $\delta$ be such that $\delta \leq \rho / 4$ with $\rho$ the gating threshold, and let $\mathbb{X}_{1}, \ldots, \mathbb{X}_{G}$ be any partition of the state space $\mathbb{X}$ satisfying properties (40)-(41). Then, the approximation error between $v_{g}^{i}(x)$ and $\hat{v}_{g}^{i}(x)$ can be upper-bounded as follows

$$
\left\|v_{g}^{i}(x)-\hat{v}_{g}^{i}(x)\right\|_{1} \leq \mu^{i}[1-F(\delta, \operatorname{dim}(x))]
$$

where $\mu^{i}$ is the estimated number of targets

$$
\mu^{i}=\sum_{p=1}^{J^{i}} \alpha_{p}^{i}
$$

Proof: see Appendix A.

Notice that the approximation error tends to vanish as $\delta$ (and hence $\rho$ ) increases, because the portion of each Gaussian $\mathcal{G}_{p}^{i}$ with $(i, p) \in \mathcal{C}_{g}$ contained in the corresponding subset $\mathbb{X}_{g}$ increases. In fact, a large $\rho$ implies that GCs belonging to different clusters are well separated. In the limiting case, the approximation error can be neglected if all clusters are wellseparated. On the other hand, when $\rho$ is large a sub-intensity function may not necessarily correspond to only one target, but might also represent multiple closely-spaced targets.

\section{Performance eValuation}

In this section, the proposed robust distributed fusion with unknown multiple FoVs is applied to both CPHD and PHD filters and the resulting DMTT performance is evaluated by means of simulation experiments in terms of the Optimal SubPattern Assignment (OSPA) metric (with Euclidean distance $c=600[m]$, and cutoff parameter $p=1$ ) [45].

The target state is defined as $x_{k}=\left[p_{x, k}, \dot{p}_{x, k}, p_{y, k}, \dot{p}_{y, k}\right]^{\top}$, where $\left(p_{x, k}, p_{y, k}\right)$ and $\left(\dot{p}_{x, k}, \dot{p}_{y, k}\right)$ are the Cartesian coordinates of position and velocity, respectively. The state transition density, for each target, is assumed to be linear Gaussian, i.e.,

$$
f\left(x_{k} \mid x_{k-1}\right)=\mathcal{G}\left(x_{k} ; A x_{k-1}, Q\right),
$$

with

$$
A=\left[\begin{array}{cc}
1 & T_{s} \\
0 & 1
\end{array}\right] \otimes I_{2}, Q=\sigma_{w}^{2}\left[\begin{array}{cc}
\frac{T_{s}^{4}}{4} & \frac{T_{s}^{3}}{2} \\
\frac{T_{s}^{3}}{2} & T_{s}^{2}
\end{array}\right] \otimes I_{2},
$$

where $I_{n}$ denotes the $n \times n$ identity matrix and $T_{s}$ is the sampling interval. $A$ and $Q$ are the state transition matrix and process noise covariance with $\sigma_{w}=5\left[\mathrm{~ms}^{-1}\right]$, respectively.

Each node deployed in the sensor network is able to provide both Time-of-Arrival (TOA) and Direction-of-Arrival (DOA) measurements, which are characterized by the following observation model:

$$
z_{k}^{i}=\left[\begin{array}{c}
\operatorname{atan} 2\left(p_{x, k}-p_{x}^{i}, p_{y, k}-p_{y}^{i}\right) \\
\sqrt{\left(p_{x, k}-p_{x}^{i}\right)^{2}+\left(p_{y, k}-p_{y}^{i}\right)^{2}}
\end{array}\right]+\varepsilon_{k},
$$

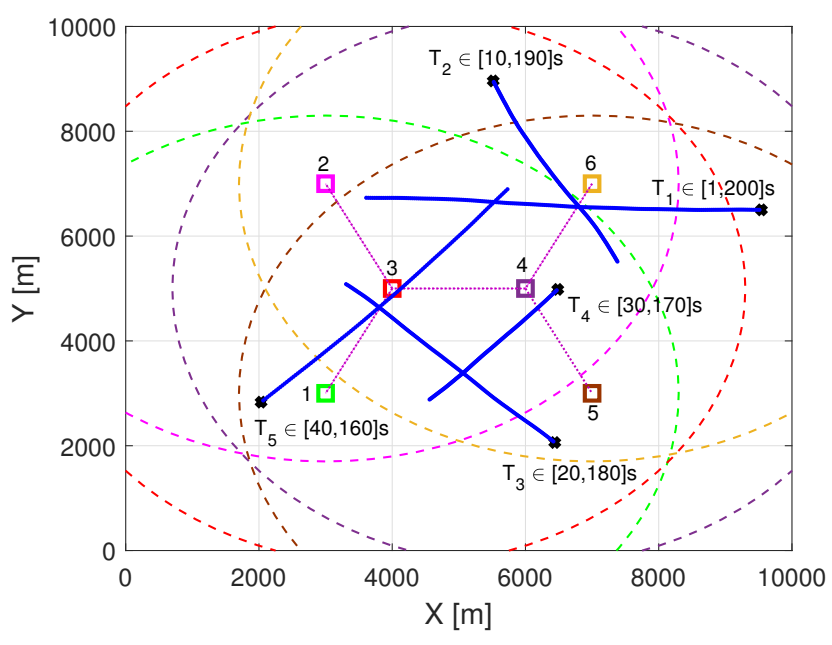

Fig. 2. Target trajectories and sensor network considered in the simulated scenario, where the circles with different colors represent different nodes and the dashed lines of the same colors depict the corresponding FoVs. ' $\square$ ' is the position of node and ' $x$ ' denotes the start point for each trajectory.

where $\left(p_{x}^{i}, p_{y}^{i}\right)$ denotes the known position of node $i$. The covariance of the measurement noises is

$$
R=\operatorname{diag}\left(\left[\sigma_{\theta}^{2}, \sigma_{r}^{2}\right]^{\top}\right)
$$

with $\sigma_{r}=5[m]$ and $\sigma_{\theta}=1\left[^{\circ}\right]$.

Clutter, for each node, is modeled as a Poisson RFS with an intensity of $\lambda_{c}=15$, which means that there are, on average, 15 clutter points per scan. The survival probability for each target is $p_{S}=0.95$. Each node has a limited FoV as depicted in Fig. 2 The probability of detection within the FoV is considered constant equal to $p_{D, 0} \in(0,1)$, while it is zero outside the FoV. Thus, the detection probability for any node $i$ is modeled as follows.

$$
p_{D}^{i}(x)=p_{D, 0} 1_{\mathcal{S}^{i}}(x) .
$$

Further, the adaptive birth model based on received measurements is adopted as in [46]. Each measurement is regarded as a potentially newborn target with

$$
\begin{aligned}
& m_{\gamma, k}(1)=z_{k}(2) \sin \left(z_{k}(1)\right), \\
& m_{\gamma, k}(3)=z_{k}(2) \cos \left(z_{k}(1)\right),
\end{aligned}
$$

and $m_{\gamma, k}(2)=m_{\gamma, k}(4)=0, P_{b}=\operatorname{diag}\left([50,20,50,20]^{\top}\right)^{2}$. The average number of newborn targets per frame is taken as 0.15 . Further, the cardinality distribution of newborn targets is assumed Poisson. The maximum number of Gaussian components is set to 40 and the maximum number of targets to 20. A consensus-based distributed fusion architecture is considered wherein each sensor node fuses its local posterior with those of its neighbors. More specifically, in each sampling interval, information exchange and fusion are repeated 3 times, corresponding to 3 consensus steps.

To assess the performance of the proposed distributed fusion with multiple FoVs, 100 Monte Carlo (MC) runs are performed on the same trajectories but with independently generated measurements (including clutter and target-generated 


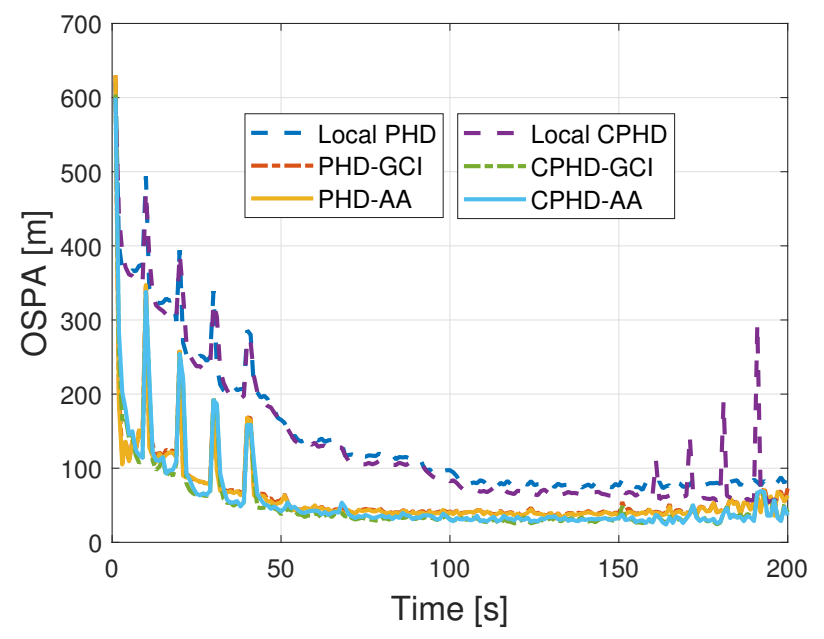

Fig. 3. Comparison of OSPA errors between local PHD, local CPHD, PHDGCI, PHD-AA, CPHD-GCI, and CPHD-AA under high detection probability.

measurements) for each trial.

\section{A. Scenario 1}

Filtering with high detection probability $\left(p_{D, 0}=0.95\right)$ is considered here. The comparisons of OSPA errors and cardinality estimates are respectively shown in Figs. 3 and 4 For both PHD and CPHD filters, the performance of GCI and AA fusion are almost the same. Moreover, the performance of CPHD-based fusion is better than PHD-based one, even if the gap is not very large. It can also be seen that, after the transient, CPHD-GCI performs slightly better than CPHD-AA. Moreover, the filtering performance of single node PHD/CPHD is very poor, especially in the initial stage of filtering. This is because targets do not fully enter the $\mathrm{cFoV}$ of all nodes.

On the other hand, the cardinality comparison shown in Fig. 4 shows that the target number estimates of local PHD/CPHD filters are very poor before all targets enter the $\mathrm{cFoV}$. It can also be noticed that all fusion approaches provide good results and much better than local PHD/CPHD filters.

\section{B. Scenario 2}

In this case, lower detection probability is considered, i.e. $p_{D, 0}=0.65$. OSPA errors and cardinality estimates are respectively given in Figs. 5 and 6 It is evident that CPHDAA and CPHD-GCI outperform PHD-AA and PHD-GCI, respectively. Meanwhile, it can be found that PHD-based fusion is more sensitive to misdetection than CPHD-based one. It also turns out that, in this setting, CPHD-AA fusion provides better performance than CPHD-GCI fusion. This is due to the multiplicative nature of the GCI fusion rule by which any missed target or existing target with an extremely small weight in a local CPHD filter will reduce the weight of this target in the other nodes, thus reducing the possibility of being detected. This problem is also reflected by the cardinality underestimation of CPHD-GCI fusion shown in Fig. 6.

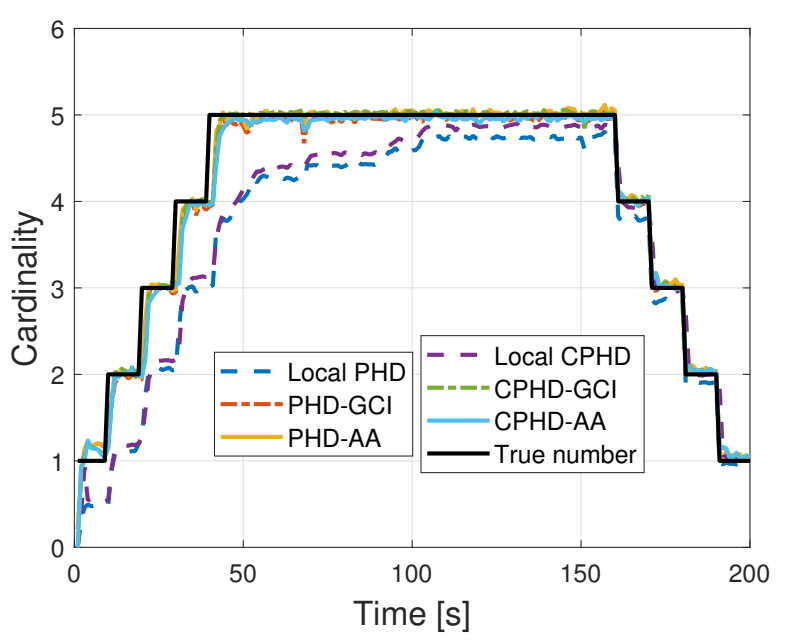

Fig. 4. Comparison of cardinality estimates between local PHD, local CPHD, PHD-GCI, PHD-AA, CPHD-GCI, and CPHD-AA under high detection probability.

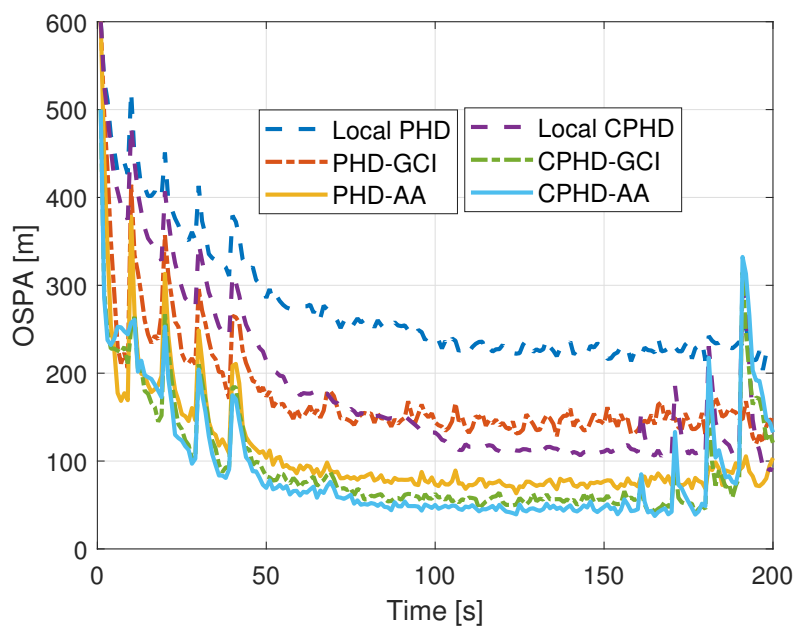

Fig. 5. Comparison of OSPA errors between local PHD, local CPHD, PHDGCI, PHD-AA, CPHD-GCI, and CPHD-AA under low detection probability.

Next, we test the average OSPA errors under different detection probabilities and the same clutter rate. The number of consensus steps of all fusion approaches is set to $L=3$. The corresponding result is illustrated in Fig. 77 It can be seen that CPHD-based fusion is always better than PHD-based fusion and that PHD-GCI fusion is more sensitive to misdetection than CPHD-GCI fusion. Furthermore, CPHD-GCI fusion outperforms CPHD-AA fusion under high detection probability while the converse is true under low detection probability. Summarizing, CPHD-AA fusion has higher tolerance to misdetection than CPHD-GCI.

\section{CONCLUSIONS}

In this paper, we have proposed a distributed multi-target tracking approach based on CPHD filtering under multiple fields-of-view (FoVs). First, it has been pointed out how standard fusion is infeasible when the fusing nodes have 


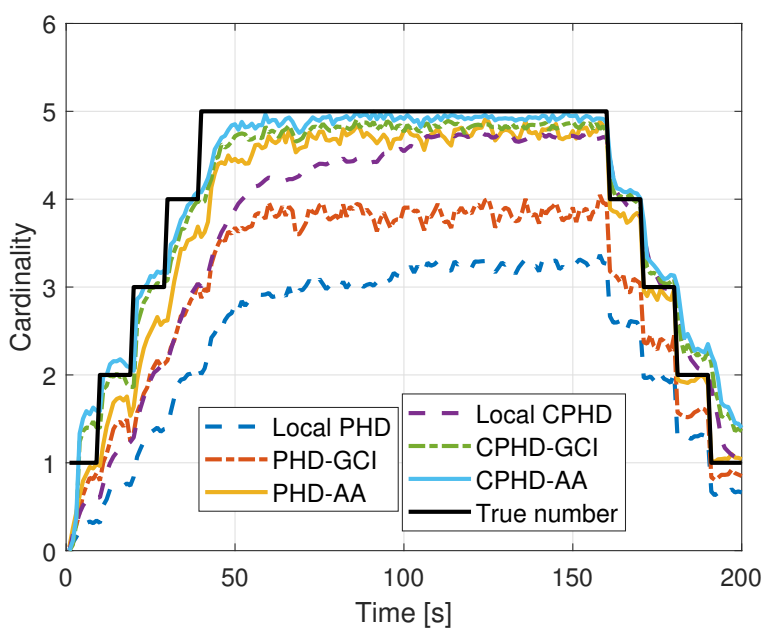

Fig. 6. Comparison of cardinality estimates between local PHD, local CPHD, PHD-GCI, PHD-AA, CPHD-GCI, and CPHD-AA under low detection probability.

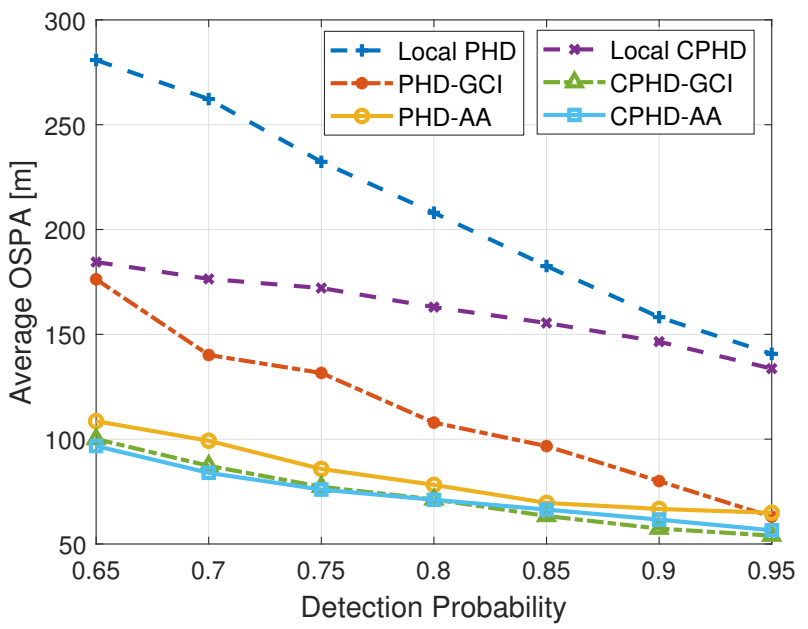

Fig. 7. Comparison of average OSPA errors between local PHD, local CPHD, PHD-GCI, PHD-AA, CPHD-GCI, and CPHD-AA under different detection probabilities.

different FoVs, and it has also been shown that the seemingly reasonable strategy of fusing information within the common FoV while copying information from the exclusive FoVs is non robust with respect to unavoidable uncertainties on the FoVs. Next, we have presented a clustering-based approach to decompose the intensity functions to be fused into multiple clusters. By approximating the target IID cluster process as multi-Bernoulli RFS, the cardinality distribution of each cluster can be re-constructed. Then, we can perform fusion for all clusters in parallel, exploiting either GCI or AA rules. Finally, all clusters are merged into an IID cluster process. Moreover, an interpretation of the proposed clustering-based fusion strategy in terms of decomposition of the state space has been provided, and an analysis of the approximation errors implied by the considered decomposition procedure has been carried out. Finally, simulation experiments have been provided to demonstrate the effectiveness of the proposed approach.

\section{ACKNOWLEDGEMENT}

We would like to thank the support of the Scholarship of China Scholarship Council (CSC).

\section{APPENDIX A PROOFS}

Proof of Lemma 1: Let $(i, p)$ and $(j, q)$ belong to different clusters. Then, we have

$$
\left(m_{p}^{i}-m_{q}^{j}\right)^{\top}\left(P_{p}^{i}+P_{q}^{j}\right)^{-1}\left(m_{p}^{i}-m_{q}^{j}\right)>\rho .
$$

Consider now the two ellipsoids $\mathcal{E}_{p}^{i}(\delta)$ and $\mathcal{E}_{q}^{j}(\delta)$ and suppose that they are not disjoint, i.e. there exists $x \in \mathbb{X}$ such that the following two conditions hold

$$
\begin{aligned}
& \left(x-m_{p}^{i}\right)^{\top}\left(P_{p}^{i}\right)^{-1}\left(x-m_{p}^{i}\right) \leq \delta, \\
& \left(x-m_{q}^{j}\right)^{\top}\left(P_{q}^{j}\right)^{-1}\left(x-m_{q}^{j}\right) \leq \delta .
\end{aligned}
$$

In turn, these conditions imply that

$$
\begin{aligned}
& \left(x-m_{p}^{i}\right)^{\top}\left(P_{p}^{i}+P_{p}^{j}\right)^{-1}\left(x-m_{p}^{i}\right) \leq \delta, \\
& \left(x-m_{q}^{j}\right)^{\top}\left(P_{p}^{i}+P_{q}^{j}\right)^{-1}\left(x-m_{q}^{j}\right) \leq \delta,
\end{aligned}
$$

because $P_{p}^{i}$ and $P_{q}^{j}$ are positive definite. Then, we can bound the corrected Mahalanobis distance as

$$
\begin{aligned}
& \left(m_{p}^{i}-m_{q}^{j}\right)^{\top}\left(P_{p}^{i}+P_{q}^{j}\right)^{-1}\left(m_{p}^{i}-m_{q}^{j}\right) \\
& =\left(m_{p}^{i}-x+x-m_{q}^{j}\right)^{\top}\left(P_{p}^{i}+P_{q}^{j}\right)^{-1}\left(m_{p}^{i}-x+x-m_{q}^{j}\right) \\
& \leq 2\left(m_{p}^{i}-x\right)^{\top}\left(P_{p}^{i}+P_{q}^{j}\right)^{-1}\left(m_{p}^{i}-x\right) \\
& \quad+2\left(x-m_{q}^{j}\right)^{\top}\left(P_{p}^{i}+P_{q}^{j}\right)^{-1}\left(x-m_{q}^{j}\right) \\
& \leq 4 \delta .
\end{aligned}
$$

Clearly, this bound is not in contradiction with (48) only if $4 \delta>\rho$. This means that when $\delta \leq \rho / 4$ the two ellipsoids $\mathcal{E}_{p}^{i}(\delta)$ and $\mathcal{E}_{q}^{j}(\delta)$ have to be disjoint or otherwise we get into a contradiction.

Proof of Theorem 1: Recalling (44) and applying the triangular inequality, we obtain

$$
\begin{aligned}
\left\|v_{g}^{i}(x)-\hat{v}_{g}^{i}(x)\right\|_{1} \leq & \sum_{p:(i, p) \in \mathcal{C}_{g}} \alpha_{p}^{i}\left\|\left[1-1_{\mathbb{X}_{g}}(x)\right] \mathcal{G}_{p}^{i}(x)\right\|_{1} \\
& +\sum_{p:(i, p) \notin \mathcal{C}_{g}} \alpha_{p}^{i}\left\|1_{\mathbb{X}_{g}}(x) \mathcal{G}_{p}^{i}(x)\right\|_{1} .
\end{aligned}
$$

Consider first a generic element of the first summation. Since $(i, p) \in \mathcal{C}_{g} \Longrightarrow \mathcal{E}_{p}^{i}(\delta) \subseteq \mathbb{X}_{g}$, we have

$$
\begin{aligned}
\left\|\left[1-1_{\mathbb{X}_{g}}(x)\right] \mathcal{G}_{p}^{i}(x)\right\|_{1} & =\int_{\mathbb{X}}\left[1-1_{\mathbb{X}_{g}}(x)\right] \mathcal{G}_{p}^{i}(x) d x \\
& =1-\int_{\mathbb{X}_{g}} \mathcal{G}_{p}^{i}(x) d x \\
& \leq 1-\int_{\mathcal{E}_{p}^{i}(\delta)} \mathcal{G}_{p}^{i}(x) d x \\
& =1-F(\delta, \operatorname{dim}(x)) .
\end{aligned}
$$


Consider now a generic element of the second summation. Since $(i, p) \notin \mathcal{C}_{g} \Longrightarrow \mathcal{E}_{p}^{i}(\delta) \cap \mathbb{X}_{g}=\emptyset$, we have

$$
\begin{aligned}
\left\|1_{\mathbb{X}_{g}}(x) \mathcal{G}_{p}^{i}(x)\right\|_{1} & =\int_{\mathbb{X}_{g}} \mathcal{G}_{p}^{i}(x) d x \\
& =1-\int_{\mathbb{X}_{\backslash \mathbb{X}}} \mathcal{G}_{p}^{i}(x) d x \\
& \leq 1-\int_{\mathcal{E}_{p}^{i}(\delta)} \mathcal{G}_{p}^{i}(x) d x \\
& =1-F(\delta, \operatorname{dim}(x)) .
\end{aligned}
$$

Combining the obtained bounds, we get

$$
\left\|v_{g}^{i}(x)-\hat{v}_{g}^{i}(x)\right\|_{1} \leq \sum_{p=1}^{J^{i}} \alpha_{p}^{i}[1-F(\delta, \operatorname{dim}(x))]
$$

from which the theorem statement follows.

\section{REFERENCES}

[1] A. Farina and F. A. Studer, Radar data processing: introduction and tracking. Letchford, UK: Research Studies Press, 1985.

[2] Y. Bar-Shalom and T. Fortmann, Tracking and data association. New York, NY, USA: Academic, 1988.

[3] S. S. Blackman and R. Popoli, Design and analysis of modern tracking systems. Norwood, MA, USA: Artech House, 1999.

[4] Y. Bar-Shalom, X. R. Li, and T. Kirubarajan, Estimation with applications to tracking and navigation. New York: Wiley, USA, 2001.

[5] R. Mahler, Advances in statistical multisource-multitarget information fusion. Norwell, MA, USA: Artech House, 2014.

[6] R. Mahler, Statistical multisource-multitarget information fusion. Norwell, MA, USA: Artech House, 2007.

[7] R. Mahler, "Multi-target Bayes filtering via first-order multi-target moments," IEEE Trans. Aerosp. Electron. Syst., vol. 39, no. 4, pp. 1152 1178,2003

[8] B. T. Vo, B. N. Vo, and A. Cantoni, "The cardinality balanced multitarget multi-Bernoulli filter and its implementations," IEEE Trans. Signal Process., vol. 57, no. 2, pp. 409-423, 2009.

[9] B. T. Vo, B. N. Vo, and D. Suter, "Joint detection and estimation of multiple objects from image observation," IEEE Trans. Signal Process., vol. 58, no. 10, pp. 5129-5141, 2010.

[10] B. N. Vo and W. K. Ma, "The Gaussian mixture probability hypothesis density filter," IEEE Trans. Signal Process., vol. 54, no. 11, pp. 40914104, 2006

[11] B. T. Vo, B. N. Vo, and A. Cantoni, "Analytic implementations of the cardinality probability hypothesis density filter," IEEE Trans. Signal Process., vol. 55, no. 7, pp. 3553-3567, 2007.

[12] H. Sidenbladh, "Multi-target particle filtering for the probability hypothesis density," in Proc. 6th Int. Conf. Inf. Fusion, 2003, pp. 800-806.

[13] B. N. Vo, S. Singh, and A. Doucet, "Sequential Monte Carlo methods for multitarget filtering with random finite sets," IEEE Trans. Aerosp. Electron. Syst., vol. 41, no. 4, pp. 1224-1245, 2005.

[14] R. Mahler, "Optimal/robust distributed data fusion: a unified approach," in Proc. SPIE Defense and Security Symp., 2000, pp. 128-138.

[15] M. Üney, D. E. Clark, and S. J. Julier, "Distributed fusion of PHD filters via exponential mixture densities," IEEE J. Sel. Topic Signal Process. vol. 7, no. 3, pp. 521-531, 2013.

[16] G. Battistelli, L. Chisci, C. Fantacci, A. Farina, and A. Graziano, "Consensus CPHD filter for distributed multitarget tracking," IEEE J. Sel. Topic Signal Process., vol. 7, no. 3, pp. 508-520, 2013.

[17] G. Battistelli, L. Chisci, A. Farina, and B. N. Vo, "Average KullbackLeibler divergence for random finite sets," in Proc. 18th Int. Conf. Inf. Fusion, 2015, pp. 1359-1366.

[18] C. Fantacci, B.-N. Vo, B.-T. Vo, G. Battistelli, and L. Chisci, "Robust fusion for multisensor multiobject tracking," IEEE Signal Process. Lett., vol. 25, no. 5, pp. 640-644, 2018.

[19] A. K. Gostar, R. Hoseinnezhad, and A. Bab-Hadiashar, "CauchySchwarz divergence-based distributed fusion with Poisson random finite sets," in Proc. ICCAIS, 2017, pp. 117-121.

[20] L. Gao, G. Battistelli, and L. Chisci, "Multiobject fusion with minimum information loss," IEEE Signal Process. Lett., vol. 27, pp. 201-205, 2020.
[21] T. Li, J. M. Corchado, and S. Sun, "Partial consensus and conservative fusion of Gaussian mixtures for distributed PHD fusion," IEEE Trans. Aerosp. Electron. Syst., 2018.

[22] T. Li, H. Fan, J. Garcia, and J. M. Corchado, "Second-order statistics analysis and comparison between arithmetic and geometric average fusion: application to multi-sensor target tracking," Informa. Fusion, vol. 51, pp. 233-243, 2019.

[23] S. Kullback and R. A. Leibler, "On information and sufficiency," Ann. Math. Statistics, vol. 22, no. 1, pp. 79-86, 1951.

[24] G. Battistelli and L. Chisci, "Kullback-Leibler average, concensus on probability density, and distributed state estimation with guaranteed stability," Automatica, vol. 50, no. 3, pp. 707-718, 2014.

[25] G. Li, W. Yi, S. Li, B. Wang, and L. Kong, "Asynchronous multirate multi-sensor fusion based on random finite set," Signal Process. vol. 160, pp. 6113-126, 2019.

[26] W. Yi, S. Li, B. Wang, R. Hoseinnezhad, and L. Kong, "Computationally efficient distributed multi-sensor fusion with multi-Bernoulli filter," IEEE Trans. Signal Process., vol. 68, pp. 241-256, 2020.

[27] B. Wang, W. Yi, R. Hoseinnezhad, S. Li, L. Kong, and X. Yang, "Distributed fusion with multi-Bernoulli filter based on generalized covariance intersection," IEEE Trans. Signal Process., vol. 65, no. 1, pp. 242-255, 2017

[28] K. Da, T. Li, Y. Zhu, H. Fan, and Q. Fu, "Kullback-Leibler averaging for multitarget density fusion," in Proc. DCAI, 2019, pp. 253-261.

[29] L. Gao, G. Battistelli, and L. Chisci, "Fusion of labeled RFS densities with minimum information loss," arXiv:1911.01083, 2019.

[30] M. Üney, J. Houssineau, E. Delande, S. J. Julier, and D. Clark, "Fusion of finite set distributions: Pointwise consistency and global cardinality," IEEE Trans. Aerosp. Electron. Syst., 2018.

[31] T. Li, Z. Liu, and Q. Pan, "Distributed Bernoulli filtering for target detection and tracking based on arithmetic average fusion," IEEE Signal Process. Lett., vol. DOI:10.1109/1sp.2019.2950588, 2019.

[32] J. Gan, M. Vasic, and A. Martinoli, "Cooperative multiple dynamic object tracking on moving vehicles based on sequential monte carlo probability hypothesis density filter," in Proc. ITSC, 2016, pp. 2163 2170.

[33] G. Battistelli, L. Chisci, and A. Laurenzi, "Random set approach to distributed multivehicle SLAM," in Proc. 20th IFAC World Congress, vol. 50, no. 1,2017 , pp. 2457-2464.

[34] G. Li, G. Battistelli, W. Yi, and L. Kong, "Distributed multi-view fusion based on generalized covariance intersection," Signal Process., vol. 166 2019.

[35] W. Liu, Y. Chen, and H. Cui, "Multi-sensor tracking with nonoverlapping field for the GLMB filter," in Proc. IEEE Int. Conf. Control, Auto. Inf. Sci., 2017, pp. 197-202.

[36] X. Wang, A. K. Gostar, T. Rathnayake, B. Xu, A. Bab-Hadiashar, an R. Hoseinnezhad, "Centralized multiple-view sensor fusion using labeled multi-Bernoulli filters," Signal Process., vol. 150, pp. 75-84, 2018.

[37] S. Li, G. Battistelli, L. Chisci, W. Yi, B. Wang, and L. Kong, "Multisensor multi-object tracking with different fields-of-view using the LMB filter," in Proc. 21th Int. Conf. Inf. Fusion, 2018, pp. 1201-1208.

[38] T. Li, V. Elvira, H. Fan, and J. M. Corchado, "Local diffusion based distributed SMC-PHD filtering using sensors with limited sensing range," IEEE Sensors J., vol. 19, pp. 1580-1589, 2019.

[39] D. Clark, S. J. Julier, R. Mahler, and B. Ristic, "Robust multi-object sensor fusion with unknown correlations," in Proc. Sensor Signal Process. for Defence, 2010, pp. 1-5.

[40] L. Gao, G. Battistelli, L. Chisci, and P. Wei, "Distributed joint sensor registration and target tracking via sensor network," Informa. Fusion, vol. 46, pp. 218-230, 2019.

[41] X. Wang, A. K. Gostar, T. Rathnayake, B. Xu, A. Bah-Hadiashar, and R. Hoseinnezhad, "Centralized multiple-view sensor fusion using labeled multi-Bernoulli filters," Signal Process., vol. 150, pp. 75-84, 2018.

[42] O. Erdinc, P. Willett, and Y. Bar-Shalom, "The bin-occupancy filter and its connection to the PHD filters," IEEE Trans. Signal Process., vol. 57, no. 11 , pp. 4232-4246, 2019.

[43] N. I. Achieser, Theory of approximation. New York: Ungar, 1956.

[44] B. Silverman, Density estimation for statistics and data analysis. London, U.K.: Chapman Hall, 1986.

[45] D. Schuhmacher, B. T. Vo, and B. N. Vo, "A consistent metric for performance evaluation of multi-object filters," IEEE Trans. Signal Process., vol. 56, no. 8, pp. 3447-3457, 2008.

[46] B. Ristic, D. Clark, B. N. Vo, and B. T. Vo, "Adaptive target birth intensity for PHD and CPHD filters," IEEE Trans. Aerosp. Electron. Syst., vol. 48, no. 2, pp. 1656-1668, 2012. 